\title{
Photonic Crystal Fano Laser: Terahertz Modulation and Ultrashort Pulse Generation
}

\author{
Mørk, Jesper; Chen, Yaohui; Heuck, Mikkel
}

Published in:

Physical Review Letters

Link to article, DOI:

10.1103/PhysRevLett.113.163901

Publication date:

2014

Document Version

Publisher's PDF, also known as Version of record

Link back to DTU Orbit

Citation (APA):

Mørk, J., Chen, Y., \& Heuck, M. (2014). Photonic Crystal Fano Laser: Terahertz Modulation and Ultrashort Pulse Generation. Physical Review Letters, 113(16), 163901. https://doi.org/10.1103/PhysRevLett.113.163901

\section{General rights}

Copyright and moral rights for the publications made accessible in the public portal are retained by the authors and/or other copyright owners and it is a condition of accessing publications that users recognise and abide by the legal requirements associated with these rights.

- Users may download and print one copy of any publication from the public portal for the purpose of private study or research.

- You may not further distribute the material or use it for any profit-making activity or commercial gain

- You may freely distribute the URL identifying the publication in the public portal 


\title{
Photonic Crystal Fano Laser: Terahertz Modulation and Ultrashort Pulse Generation
}

\author{
J. Mork, ${ }^{*}$ Y. Chen, and M. Heuck \\ DTU Fotonik, Technical University of Denmark, DK-2800 Kongens Lyngby, Denmark
}

(Received 28 May 2014; published 15 October 2014)

\begin{abstract}
We suggest and analyze a laser with a mirror realized by Fano interference between a waveguide and a nanocavity. For small-amplitude modulation of the nanocavity resonance, the laser can be modulated at frequencies exceeding $1 \mathrm{THz}$, not being limited by carrier dynamics as for conventional lasers. For larger modulation, a transition from pure frequency modulation to the generation of ultrashort pulses is observed. The laser dynamics is analyzed by generalizing the field equation for conventional lasers to account for a dynamical mirror, described by coupled mode theory.
\end{abstract}

DOI: 10.1103/PhysRevLett.113.163901

PACS numbers: 42.55.Tv, 05.45.Xt, 42.55.Px

Defects in photonic crystals allow the realization of high quality optical cavities and have been used to demonstrate ultracompact lasers. Both zero-dimensional defect lasers, featuring ultrasmall cavity volumes [1-4], as well as linedefect lasers $[5,6]$ have been demonstrated. These novel types of lasers promise many advantages, such as ultralow power operation [6], ultrahigh speed [7], and switchable lasing [8], due to the possibilities of controlling the cavity modes and enhancing the light-matter interaction [9]. In this work we suggest a novel photonic crystal laser, illustrated in Fig. 1, which shows intriguing dynamical properties. The right mirror is formed by a Fano interference between a waveguide (continuum) mode and the localized resonance of a side-coupled nanocavity [10]. Because of destructive interference between light transmitted through the waveguide and the cavity, this structure leads to a narrow reflection band centered at the nanocavity resonance. The left mirror may be realized by simply terminating the defect waveguide; see, e.g., Ref. [11]. Open configurations, where the left and right sides of the waveguide couple via the cavity, were used to demonstrate ultracompact and low-power optical switches [12-15]. We derive a theoretical model for Fano resonance based lasers and show that these can be frequency modulated (FM) via the mirror cavity at frequencies much larger than possible in conventional semiconductor lasers. Furthermore, the laser displays rich dynamics when changing the amplitude by which the resonance is modulated, leading to a regime of operation where ultrashort pulses are generated. We show that this behavior is related to a nonlinear and asymmetric mode tuning characteristic, originating in the destruction of a mode upon tuning the cavity resonance.

The suggested structure is also of interest in fundamental investigations of the nonlinear dynamics of coupled lasers [16-18], providing a new interaction mechanism between lasers sharing a common mirror. We notice that various types of Fano asymmetry can be realized by simply adding a scattering element in the waveguide below the nanocavity [19], but here we focus on the simplest type. High-contrast gratings used as mirrors in surface emitting lasers may also be interpreted as relying on Fano effects [20,21], but in that case the mirror is very broadband and the behavior is entirely different.

The coupling between a waveguide and a cavity can be analyzed using coupled mode theory [22], resulting in the following expression for the effective reflection coefficient $r_{2}(\omega)$ :

$$
r_{2}(\omega)=-P \frac{\gamma_{C}}{i\left(\omega_{C}-\omega\right)+\gamma_{T}},
$$
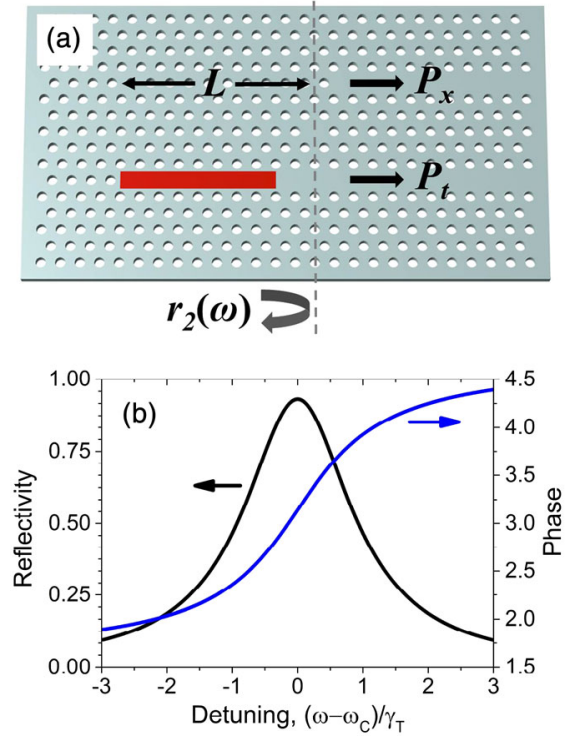

FIG. 1 (color online). (a) Schematic of line-defect photonic crystal laser structure with right mirror formed by Fano interference between the waveguide mode and a side-coupled nanocavity. The upper waveguide is not essential to laser operation but significantly increases the laser output power. (b) Effective reflection coefficient (black line) and phase (blue line) for the nanocavity mirror as seen from the waveguide. The total quality factor of the nanocavity is $Q=500$ while the internal quality factor is $Q_{i}=14300$. 
which is illustrated in Fig. 1(a). The corresponding transmission coefficient is $t_{2}(\omega)=1-\operatorname{Pr}_{2}(\omega)$. Here, $\omega_{C}$ is the cavity resonance frequency and $P$ is the cavity parity, with $P=1(-1)$ for a mode that is even (odd) with respect to the vertical nanocavity mirror symmetry line [dashed line in Fig. 1(a)]. Furthermore, $\gamma_{T}$ is the total cavity decay rate, $\gamma_{T}=\gamma_{C}+\gamma_{i}$, with $\gamma_{C}$ being the cavity waveguide coupling rate, and $\gamma_{i}=\gamma_{i^{\prime}}+\gamma_{P} / 2$ the intrinsic decay rate of the cavity, including out-of-plane scattering loss, with rate $\gamma_{i^{\prime}}$, and decay into other waveguides, with rate $\gamma_{P}$ (upper waveguide in Fig. 1). The corresponding quality factors are given as $Q_{x}=\omega_{0} / 2 \gamma_{x}$.

Introducing the left mirror reflection coefficient $r_{1}(\omega)$, the laser oscillation condition may be expressed as

$$
r_{1}(\omega) r_{2}(\omega) \exp \{2 i k(\omega, N) L\}=1,
$$

where $L$ is the laser cavity length and $k(\omega, N)$ is the complex wave number depending on frequency and carrier density $N$ [23],

$$
k(\omega, N)=\frac{\omega}{c} n(\omega, N)-i \frac{1}{2}\left[\Gamma g(\omega, N)-\alpha_{i}\right] .
$$

Here, $n$ is the refractive index, $\Gamma$ is the confinement factor, $\alpha_{i}$ is the internal waveguide loss, and $g(\omega, N) \simeq g_{N}\left(N-N_{0}\right)$ is the material gain, with $g_{N}$ being the differential gain and $N_{0}$ the carrier density at transparency.

We now assume that for a given reference laser cavity length, $L=L_{r}$, the laser oscillates at the reference frequency $\omega_{r}$, which coincides with the mirror resonance frequency, $\omega_{C}$. Employing linear expansions of gain and refractive index, we calculate the variation of the laser frequency $\omega_{s}$ and threshold gain $g_{\text {th }}$ when detuning the cavity resonance frequency from the reference frequency by numerical solution of Eq. (2); see Fig. 2.

The increase of threshold gain upon detuning the cavity resonance frequency occurs since the oscillation (phase) condition for the laser frequency is not fulfilled at the detuned cavity frequency. The laser thus oscillates off the mirror resonance, such that the phase imparted by the mirror, cf. Fig. 1(b), ensures the fulfilment of the phase condition. The asymmetry of the threshold gain with respect to detuning originates from the finite value of the alpha parameter [23]. Upon red-tuning the cavity, the phase change due to the larger carrier density thus forces the laser to oscillate further off-resonance and eventually the laser mode ceases to exist. Mathematically, this happens by the annihilation of the stable mode by collision with an unstable mode; see, e.g., Ref. [24]. A dynamical model for the laser is derived by using the general approach of Ref. [23]. Defining the right, $A^{+}(t)$, and left, $A^{-}(t)$, propagating fields left of the reference plane [dashed line in Fig. 1(a)], dynamical equations are derived by Taylor expansion around the stationary solution. The dynamics of the field stored in the nanocavity is then naturally
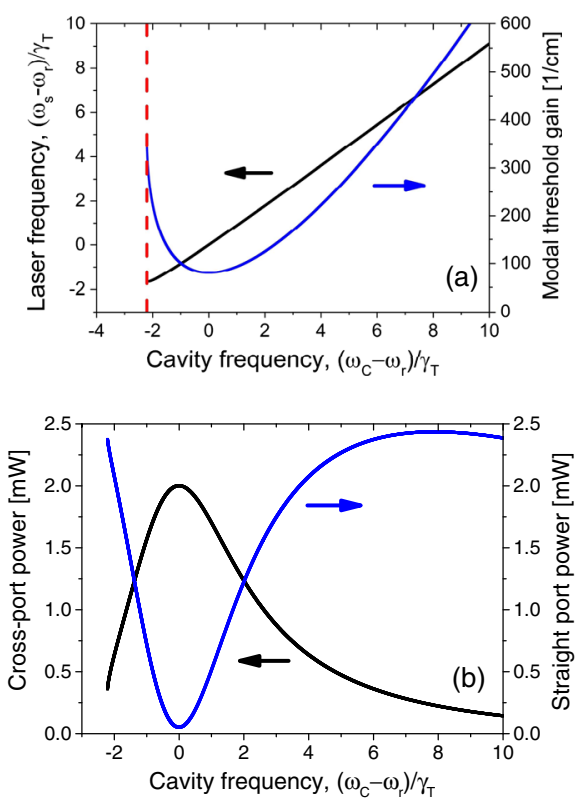

FIG. 2 (color online). Variation of (a) laser frequency and threshold gain and (b) throughport $P_{t}$, and crossport $P_{x}$ power as a function of nanocavity resonance frequency. Nonlinear mirror cavity effects are neglected.

incorporated via temporal coupled mode theory $[15,19,25]$. By neglecting the frequency dependence of the gain and the left mirror as well as spontaneous emission, we get

$$
\begin{aligned}
\frac{d A^{+}}{d t}= & \frac{1}{2}(1-i \alpha) \Gamma v_{g} g_{N}\left(N(t)-N_{s}\right) A^{+}(t) \\
& +\gamma_{L}\left[A^{-}(t) / r_{2}\left(\omega_{r}\right)-A^{+}(t)\right] . \\
\frac{d A^{-}}{d t} & =\left(-i \delta_{C}-\gamma_{T}\right) A^{-}(t)-P \gamma_{C} A^{+}(t) .
\end{aligned}
$$

Here, $N_{s}$ is the steady-state carrier density, $\gamma_{L}=c /\left(2 n_{g} L\right)$ is the inverse of the cavity round-trip time, and $\delta_{C}=$ $\omega_{C}-\omega_{r}+\Delta \omega_{\mathrm{NL}}$ is the detuning of the mirror cavity resonance from the reference frequency, $\omega_{r}$. The field $A^{-}(t)$ is related to the nanocavity field, $a(t)$, as $A^{-}(t)=$ $s_{1}^{-}(t)=-P \sqrt{\gamma_{C} /\left(2 \epsilon_{0} n c\right)} a(t)$, upon use of which, Eq. (5) reduces to the well-known equation for $a(t)$ [25].

The carrier density in the active region is governed by the standard rate equation

$$
\frac{d N}{d t}=\frac{J}{e V_{C}}-R(N)-v_{g} g(N) \frac{I(t)}{V_{p}} .
$$

Here, $I(t)$ is the photon number, $V_{C}$ is the active region volume, $V_{P}=V_{C} / \Gamma$ is the modal volume, $J$ is the current, and $R(N)$ is the recombination rate, often approximated as $R(N) \simeq N / \tau_{S}$, with $\tau_{S}$ being the carrier lifetime. The photon number is given as $I(t)=\sigma_{s}\left|A^{+}(t)\right|^{2}$, with 
$\sigma_{s}=\left(2 \epsilon_{0} n n_{q}\right) /\left(\hbar \omega_{s}\left(\Gamma g_{s}-\alpha_{i}\right)\right)\left(\left|r_{1}\right|+\left|r_{2}\right|\right)\left(1-\left|r_{1}\right|\left|r_{2}\right|\right) /$ $\left|r_{1}\right|$, where $r_{i}$ are evaluated at steady state [23]. The power transmitted in the waveguide past the nanocavity mirror (throughport) is given by $P_{t}=2 \epsilon_{0} n c\left|A^{+}(t)+P A^{-}(t)\right|^{2}$ and the power coupled to the cross-port is $P_{x}=$ $2 \epsilon_{0} n c\left(\gamma_{P} / \gamma_{C}\right)\left|A^{-}(t)\right|^{2}$. Here, $n_{g}$ is the group refractive index, taken equal to 3.5 .

The term $\Delta \omega_{\mathrm{NL}}$ accounts for nonlinear shifts of the cavity resonance due to Kerr effects as well as dispersion of carriers generated by two-photon absorption [12-15], and is included for consistency but does not qualitatively affect the results. The corresponding loss rates are represented by the imaginary part of $\Delta \omega_{\mathrm{NL}}$. We follow the detailed model given in Ref. [15] and use the parameter values quoted there, except the decay of the free carrier density is given by a single time constant of $20 \mathrm{ps}$.

Figure 2(b) shows the variation of $P_{t}$ and $P_{x}$ upon detuning the cavity. Here and in the following we use the parameter values: $L=L_{r}=5 \mu \mathrm{m}, r_{1}=1, Q_{P}=10000$, $g_{N}=5 \times 10^{-21} \mathrm{~m}^{2}, \tau_{S}=0.5 \mathrm{~ns}, \alpha_{i}=10 \mathrm{~cm}^{-1}, \alpha=1$, and $\Gamma=0.5$. When the laser oscillation frequency coincides with the mirror resonance, the power coupled out via the throughport, $P_{t}$ is very small, due to the destructive interference between the waveguide and the mirror cavity fields. On the other hand, the power coupled out via the cross-port is at its maximum, since that power is linked directly to the energy stored in the nanocavity. The laser structure may be simplified by removing the upper port, in which case low-threshold lasing can still be achieved, but the majority of the power is in this case coupled to the surroundings via the out-of-plane leakage of the nanocavity. For laser oscillation at the mirror resonance, we may derive the following approximate expression $P_{j}=$ $\eta_{0} \eta_{j}\left(J-J_{\text {th }}\right)(j=t, x)$, with $\eta_{0}=\left(\hbar \omega_{s} / e\right)\left(\Gamma g_{s}-\alpha_{i}\right) / \Gamma g_{s}$, $\eta_{t}=Q /\left(2 Q_{i}\right), \eta_{x}=Q_{i^{\prime}} /\left(Q_{i^{\prime}}+2 Q_{P}\right)$ and $J_{\text {th }}$ being the threshold current at the reference point. It was assumed that $\left|r_{1}\right|^{2} \simeq 1$ and $Q_{i} \gg Q$. We see that for $Q_{i^{\prime}} \gg Q_{P}\left(\gamma_{P} \gg \gamma_{i}^{\prime}\right)$, the differential quantum efficiency of the crossport output is only limited by internal losses. On the other hand, the throughport power is very small, scaling as $Q / Q_{i}$. We shall see later, though, that upon large-signal modulation of the mirror resonance, most of the energy stored in the laser cavity can be "dumped" via the throughport on a very short time scale.

We notice that the threshold current depends sensitively on the cavity length due to the variation of the mirror phase with frequency; cf. Fig. 1(b). Also, in order to avoid lasing at the edge of the Brillouin zone, where strong feedback occurs [26]), the nanocavity frequency should be designed to be in the fast-light regime of the waveguide.

The laser dynamics is analyzed by numerically solving Eqs. (4)-(6). We consider the case of dynamically modulating the resonance frequency of the nanocavity composing the mirror. This may be done electrically via electrodes placed in the vicinity of the nanocavity [27] and may also be experimentally explored using near-field probes which modify the resonance frequency [28]. The resonance frequency may also be changed via the dispersion of free carriers that are excited optically in the nanocavity region $[14,15,29]$, but the lifetime of those carriers will limit the laser dynamics. Using a liquid crystal infiltrated structure, switching between different modes of a photonic crystal structure was also accomplished by optical excitation [30]. We assume the nanocavity region to be passive; i.e., the active gain region is confined to the waveguide, e.g., using buried heterostructure technology [6].

We now consider the case of modulating the nanocavity resonance frequency harmonically at frequency $f$, i.e., $\omega_{C}(t)=\omega_{r}+\epsilon \gamma_{T} \cos \left(2 \pi f_{\text {mod }} t\right)$. Figure 3 shows results for constant modulation frequency, $f_{\text {mod }}=1 \mathrm{GHz}$ and different amplitudes. For $\epsilon=0.5$ (black solid line) the power $P_{t}$ varies nearly harmonically in response to the harmonic modulation of the nanocavity resonance frequency (shown as a black dashed line). When further increasing the modulation amplitude, the laser output power starts to spike, Fig. 3 (blue solid line, $\epsilon=1.8$ ), eventually leading to the generation of optical pulses (red solid line, $\epsilon=2$ ), which in the present case have a width of approximately 3 ps (FWHM). The pulse generation mechanism can be explained in analogy to $Q$ switching [31]. In our case, the laser phase condition plays a crucial role: When the mirror resonance becomes sufficiently detuned from the optimum condition, where a longitudinal mode coincides with the mirror resonance, the laser is forced below threshold and the rate of stimulated emission becomes zero. The active region electron density then starts to increase and a large electron population is built up in the laser cavity. This energy may be released when the laser passes threshold again. Since the threshold gain increases drastically for negative frequency detunings, the turn-off of the laser is first observed for negative detunings.

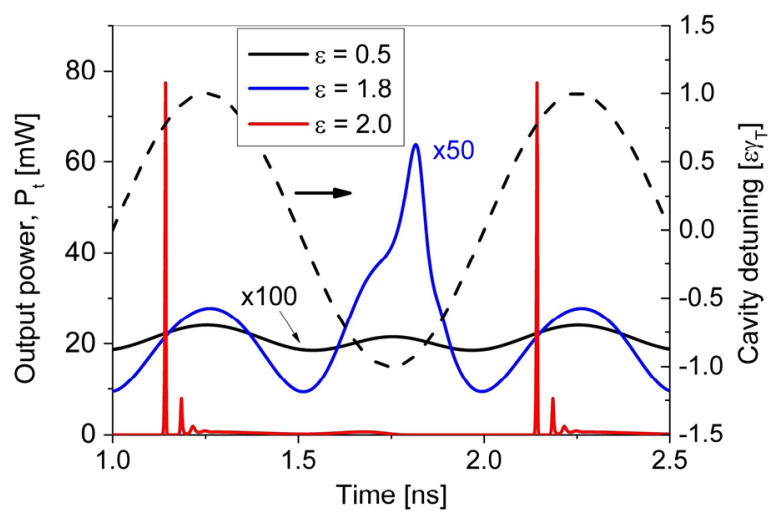

FIG. 3 (color online). Temporal variation of power coupled out via throughport $P_{t}$ for fixed modulation frequency $f_{\text {mod }}=1 \mathrm{GHz}$, fixed detuning, $\omega_{C}=\omega_{r}$, and different resonance frequency modulation amplitudes: (a) $\epsilon=0.5$ (black), (b) $\epsilon=1.8$ (blue), and (c) $\epsilon=2$ (red). The instantaneous frequency (in units of $\epsilon \gamma_{T}$, right $y$ axis) is shown as a dashed line. 
We find that the temporal position at which the pulse is emitted relative to the harmonic modulation signal changes with modulation amplitude $\epsilon$. This behavior reflects the time required for the nanocavity to reenter the abovethreshold regime, as well as the time taken for the buildup of the laser cavity field. The laser dynamics does not change qualitatively if nonlinear effects in the mirror are neglected. However, the absolute power level increases somewhat due to the absence of free-carrier and two-photon absorption and trailing pulses are further suppressed.

In Fig. 4 we investigate the regime where the modulation amplitude is smaller than the bandwidth of the resonance. The figure shows the simulated FM amplitude (circle markers, left axis), i.e., the maximum excursion of the instantaneous optical frequency and the corresponding power amplitude (triangles, right axis). The latter is quantified by the modulation index, defined as the maximum power fluctuation relative to the mean power. For $J / J_{\text {th }}=2$, the modulation index is of the order of a few percent or smaller and the crossport signal is close to a pure FM signal. The most striking result of Fig. 4 is that the laser can be modulated at very high frequencies; i.e., the 3-dB modulation bandwidth is as high as $1.5 \mathrm{THz}$, orders of magnitude higher than the bandwidth of conventional lasers [33]. Figure 4 (black solid line) also shows an analytical result for the response obtained by neglecting intensity fluctuations, which is seen to agree very well with the numerically calculated FM amplitude for the lower current. From that response we can express the 3-dB frequency modulation bandwidth as $f_{3 \mathrm{~dB}}=\left(\gamma_{L}+\gamma_{T}\right) / 2 \pi$. For the considered example: $\gamma_{L} / 2 \pi=1.34 \mathrm{THz}(5 \mu \mathrm{m}$ long cavity, $\left.n_{g}=3.5\right)$ and $\gamma_{T} / 2 \pi=194 \mathrm{GHz}(Q=500)$, resulting in a modulation bandwidth of $1.53 \mathrm{THz}$.

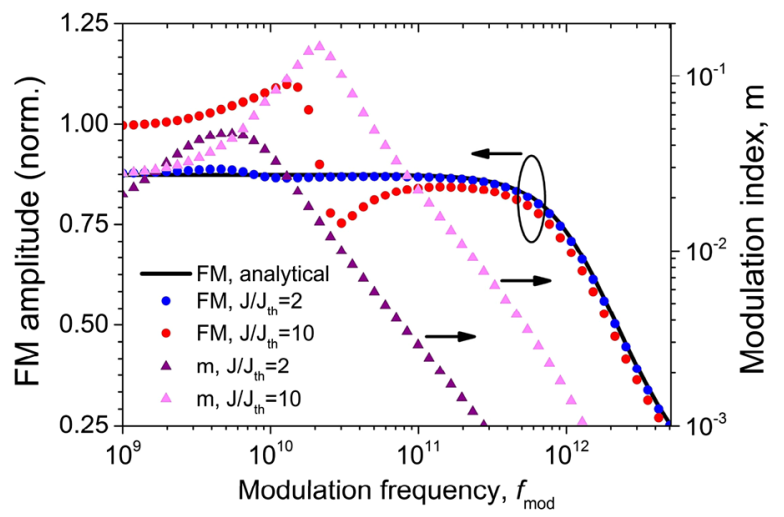

FIG. 4 (color online). FM modulation amplitude (left axis) relative to mirror resonance modulation amplitude $\epsilon \gamma_{T}$ vs frequency for $J / J_{\text {th }}=2$ (blue circles) and $J / J_{\text {th }}=10$ (red circles) and the analytical result (black solid line). Power modulation index (right axis) for the throughport for $J / J_{\text {th }}=$ 2 (purple triangles) and $J / J_{\text {th }}=10$ (magenta triangles). The modulation amplitude is fixed at $\epsilon=0.2$ and the cavity is offset by $\omega_{C}-\omega_{r}=-0.08 \gamma_{T}$ for $J / J_{\text {th }}=2$ and by $\omega_{C}-\omega_{r}=-0.82 \gamma_{T}$ for $J / J_{\text {th }}=10$.
Surprisingly, the modulation bandwidth is given by the sum of the mirror cavity decay rate and the inverse of the waveguide round-trip time, i.e., the larger of the two rates dominates, whereas usually the longest time constant governing a response limits the bandwidth. Insight into this behavior can be obtained by considering the phase difference between the coherent fields in the two cavities, $\Delta \phi(t) \equiv \arg \left\{A^{+}(t)\right\}-\arg \left\{A^{-}(t)\right\}$, which governs the laser field. Neglecting intensity fluctuations, the phase difference follows

$$
\frac{d \Delta \phi}{d t}=-\left(\gamma_{L}+\gamma_{T}\right) \Delta \phi+\epsilon \gamma_{T} \cos \left(2 \pi f_{\bmod } t\right) .
$$

The modulation of the mirror resonance thus changes the phase (and frequency) of the mirror cavity field via the adiabatic wavelength conversion mechanism [29], and since the phase difference can relax both via the waveguide cavity, governed by rate $\gamma_{L}$ and the mirror cavity, governed by $\gamma_{T}$, the characteristic relaxation rate for the phase difference is the sum of the two. Since the power and carrier density change negligibly with time, the usual limitation imposed by the relaxation oscillation frequency is absent. While the intrinsic laser modulation bandwidth thus may be even further increased by reducing the laser cavity length, as confirmed by numerical simulations, this will also increase the laser threshold gain and thus reduce the output power and increase the relative intensity noise for fixed current. Furthermore, upon electrical modulation, the bandwidth of the electrical circuitry will reduce the modulation of the resonance wavelength at high frequencies. The optimum cavity length is therefore determined, in practice, by the demands on bandwidth, power, and size. The Fabry-Perot model itself is expected to hold for laser cavity lengths down to a couple of lattice periods [11].

For $J / J_{\text {th }}=10$, both the FM modulation and the modulation index develop a resonance around $20 \mathrm{GHz}$, cf. Fig. 4, and the numerical FM response only agrees with the analytical response for frequencies beyond $100 \mathrm{GHz}$. This resonance appears since, for larger power levels, two-photon absorption leads to the generation of carriers in the cavity, which change the cavity resonance and loss. Intensity fluctuations can thus no longer be neglected and carrier dynamics in the mirror cavity and in the active region become important. The observed resonance of approximately $20 \mathrm{GHz}$ is significantly higher than the corresponding relaxation oscillation frequency, which is estimated to be $5.9 \mathrm{GHz}$. It was recently shown that a nanocavity excited by a constant optical beam may develop oscillations with a period in the 10-ps range (damped or undamped) when taking into account the carrier generation in the nanocavity [32], in qualitative agreement with the resonance observed here.

In conclusion, we have suggested and analyzed a new type of photonic crystal Fano laser which displays two 
remarkable effects. First, the laser can be frequency modulated in a bandwidth largely exceeding that of conventional lasers, enabled by adiabatic wavelength conversion and fast phase relaxation. Second, the laser may undergo a nonlinear transition to a regime where ultrashort optical pulses are generated.

This work was funded by Villum Fonden via the NATEC Centre of Excellence.

jesm@fotonik.dtu.dk

[1] O. Painter, R. K. Lee, A. Scherer, A. Yariv, J. D. O'Brien, P. D. Dapkus, and I. Kim, Science 284, 1819 (1999).

[2] H.-G. Park, S.-H. Kim, S.-H. Kwon, Y.-G. Ju, J.-K. Yang, J.-H. Baek, S.-B. Kim, and Y.-H. Lee, Science 305, 1444 (2004).

[3] T. Baba, D. Sano, K. Nozaki, K. Inoshita, Y. Kuroki, and F. Koyama, Appl. Phys. Lett. 85, 3989 (2004).

[4] D. Englund, H. Altug, B. Ellis, and J. Vuckovic, Laser Photonics Rev. 2, 264 (2008).

[5] K. Kiyota, T. Kise, N. Yokouchi, T. Ide, and T. Baba, Appl. Phys. Lett. 88, 201904 (2006).

[6] S. Matsuo, A. Shinya, T. Kakitsuka, K. Nozaki, T. Segawa, T. Sato, Y. Kawaguchi, and M. Notomi, Nat. Photonics 4, 648 (2010).

[7] H. Altug, D. Englund, and J. Vuckovic, Nat. Phys. 2, 484 (2006).

[8] S. Zhukovsky, D. Chigrin, A. Lavrinenko, and J. Kroha, Phys. Rev. Lett. 99, 073902 (2007).

[9] N. Gregersen, T. Suhr, M. Lorke, and J. Mork, Appl. Phys. Lett. 100, 131107 (2012).

[10] Z. Zhang and M. Qiu, Opt. Express 12, 3988 (2004).

[11] P. Lalanne, C. Sauvan, and J. P. Hugonin, Laser Photonics Rev. 2, 514 (2008).

[12] C. Husko, A. de Rossi, S. Combrie, Q. V. Tran, F. Raineri, and C. W. Wong, Appl. Phys. Lett. 94, 021111 (2009).

[13] K. Nozaki, T. Tanabe, A. Shinya, S. Matsuo, T. Sato, H. Taniyama, and M. Notomi, Nat. Photonics 4, 477 (2010).

[14] M. Heuck, S. Combrie, G. Lehoucq, S. Malaguti, G. Bellanca, S. Trillo, P. T. Kristensen, J. Mork, J. P. Reithmaier, and A. de Rossi, Appl. Phys. Lett. 103, 181120 (2013).

[15] Y. Yu et al., Opt. Express 21, 31047 (2013).
[16] H.-J. Wünsche, S. Bauer, J. Kreissl, O. Ushakov, N. Korneyev, F. Henneberger, E. Wille, H. Erzgräber, M. Peil, W. Elsässer, and I. Fischer, Phys. Rev. Lett. 94, 163901 (2005).

[17] S. Zhukovsky, D. Chigrin, and J. Kroha, Phys. Rev. A 79, 033803 (2009).

[18] M. C. Soriano, J. García-Ojalvo, C. R. Mirasso, and I. Fischer, Rev. Mod. Phys. 85, 421 (2013).

[19] M. Heuck, P. T. Kristensen, Yuriy Elesin, and Jesper Mørk, Opt. Lett. 38, 2466 (2013).

[20] C. Sciancalepore, B.e Ben Bakir, X. Letartre, J.-M. Fedeli, N. Olivier, D. Bordel, C. Seassal, P. Rojo-Romeo, P. Regreny, and P. Viktorovitch, J. Lightwave Technol. 29, 2015 (2011).

[21] W. Zhou, D. Zhao, Y.-C. Shuai, H. Yang, S. Chuwongin, A. Chadha, J.-H. Seo, K. X. Wang, V. Liu, Z. Ma, and S. Fan, Prog. Quantum Electron. 38, 1 (2014).

[22] S. Fan, W. Suh, and J. D. Joannopoulos, J. Opt. Soc. Am. A 20, 569 (2003).

[23] B. Tromborg, H. Olesen, X. Pan, and S. Saito, IEEE J. Quantum Electron. 23, 1875 (1987).

[24] J. Mork, B. Tromborg, and J. Mark, IEEE J. Quantum Electron. 28, 93 (1992).

[25] W. Suh, Z. Wang, and S. Fan, IEEE J. Quantum Electron. 40, 1511 (2004).

[26] M. Okano, T. Yamada, J. Sugisaka, N. Yamamoto, M. Itoh, T. Sugaya, K. Komori, and M. Mori, J. Opt. 12, 075101 (2010).

[27] L.-D. Haret, X. Checoury, F. Bayle, N. Cazier, P. Boucaud, S. Combrié, and A. de Rossi, Opt. Express 21, 10324 (2013).

[28] A. F. Koenderink, M. Kafesaki, B. C. Buchler, and V. Sandoghdar, Phys. Rev. Lett. 95, 153904 (2005).

[29] T. Tanabe, M.Notomi, H. Taniyama, and E. Kuramochi, Phys. Rev. Lett. 102, 043907 (2009).

[30] B. Maune, J. Witzens, T. Baehr-Jones, M. Kolodrubetz, H. Atwater, A. Scherer, R. Hagen, and Y. Qiu, Opt. Express 13, 4699 (2005).

[31] U. Keller and K. J. Weingarten, IEEE J. Sel. Top. Quantum Electron. 2, 435 (1996).

[32] S. Malaguti, G. Bellanca, A. de Rossi, S. Combrié, and S. Trillo, Phys. Rev. A 83, 051802 (2011).

[33] L. Coldren, S. Corzine, and M. Masanovic, Diode Lasers and Photonic Integrated Circuits (Wiley, New York, 2012). 